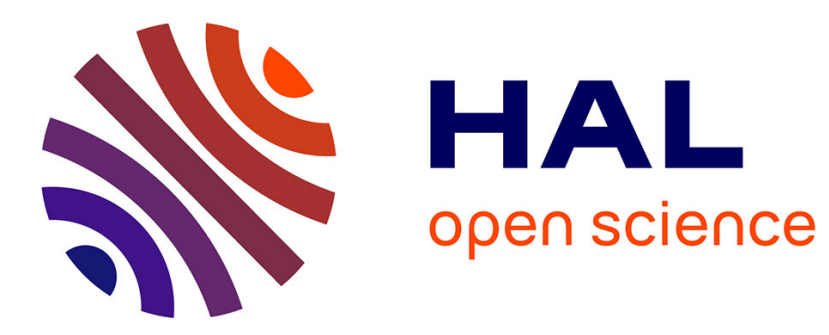

\title{
An Analysis of Decentralized Airspace Structure and Capacity Using Fast-Time Simulations
}

Emmanuel Sunil, Joost Ellerbroek, Jacco Hoekstra, Andrija Vidosavljevic, Michael Arntzen, Frank Bussink, Dennis Nieuwenhuisen

\section{- To cite this version:}

Emmanuel Sunil, Joost Ellerbroek, Jacco Hoekstra, Andrija Vidosavljevic, Michael Arntzen, et al.. An Analysis of Decentralized Airspace Structure and Capacity Using Fast-Time Simulations. Journal of Guidance, Control, and Dynamics, 2017, 40 (1), pp. 38-51. 10.2514/1.G000528 . hal-01457367

\section{HAL Id: hal-01457367 \\ https://hal-enac.archives-ouvertes.fr/hal-01457367}

Submitted on 13 Feb 2017

HAL is a multi-disciplinary open access archive for the deposit and dissemination of scientific research documents, whether they are published or not. The documents may come from teaching and research institutions in France or abroad, or from public or private research centers.
L'archive ouverte pluridisciplinaire HAL, est destinée au dépôt et à la diffusion de documents scientifiques de niveau recherche, publiés ou non, émanant des établissements d'enseignement et de recherche français ou étrangers, des laboratoires publics ou privés. 


\title{
An Analysis of Decentralized Airspace Structure and Capacity Using Fast-Time Simulations
}

\author{
${\text { Emmanuel Sunil* }{ }^{*} \text { Joost Ellerbroek }{ }^{\dagger} \text { and Jacco Hoekstra }}^{\ddagger}$ \\ Delft University of Technology (TU Delft), Delft, 2628HS, The Netherlands
}

\author{
Andrija Vidosavljevic ${ }^{\S}$ \\ Ecole Nationale de l'Aviation Civile (ENAC), Toulouse, 31055, France
}

\begin{abstract}
Michael Arntzen ${ }^{\mathbb{I}[\|}$, Frank Bussink** and Dennis Nieuwenhuisen ${ }^{\dagger \dagger}$
National Aerospace Laboratory (NLR), Amsterdam, 1059CM, The Netherlands
\end{abstract}

\begin{abstract}
The work that is presented in this paper is part of an ongoing study on the relationship between airspace structure and capacity. The present paper investigates the degree of structuring needed to maximize capacity for decentralized en-route airspace. To this end, four decentralized en-route airspace concepts, which vary in terms of the number of constrained degrees of freedom, were compared using fast-time simulations, for both nominal and non-nominal conditions. The airspace structure-capacity relationship was studied from the effect of multiple traffic demand densities on airspace metrics. The results indicated that structuring methods that over-constrained the horizontal path of aircraft reduced capacity, as traffic demand displays no predominant patterns in the horizontal dimension for decentralization. The results also showed that capacity was maximized when a vertical segmentation of airspace was used to separate traffic with different travel directions at different flight levels. This mode of structuring improved performance over completely unstructured airspace by reducing relative velocities between aircraft cruising at the same altitude, while allowing direct horizontal routes.
\end{abstract}

\footnotetext{
*PhD Candidate, Control and Simulation, Faulty of Aerospace Engineering, e.sunil@tudelft.nl

${ }^{\dagger}$ Assistant Professor, Control and Simulation, Faulty of Aerospace Engineering

${ }^{\ddagger}$ Professor, Control and Simulation, Faulty of Aerospace Engineering

$\S$ Research Associate, MAIAA-Applied Mathematics Laboratory

$\mathbb{I}_{R} \& D$ Engineer, Environment \& Policy Support (NLR)

"Assistant Professor, Aircraft Noise and Climate Effects, Faulty of Aerospace Engineering,Delft University of Technology (TU Delft)

**R\&D Engineer, Cockpit and Flight Operations

${ }^{\dagger \dagger}$ R\&D Engineer, ATM and Airports
} 


\section{Nomenclature}

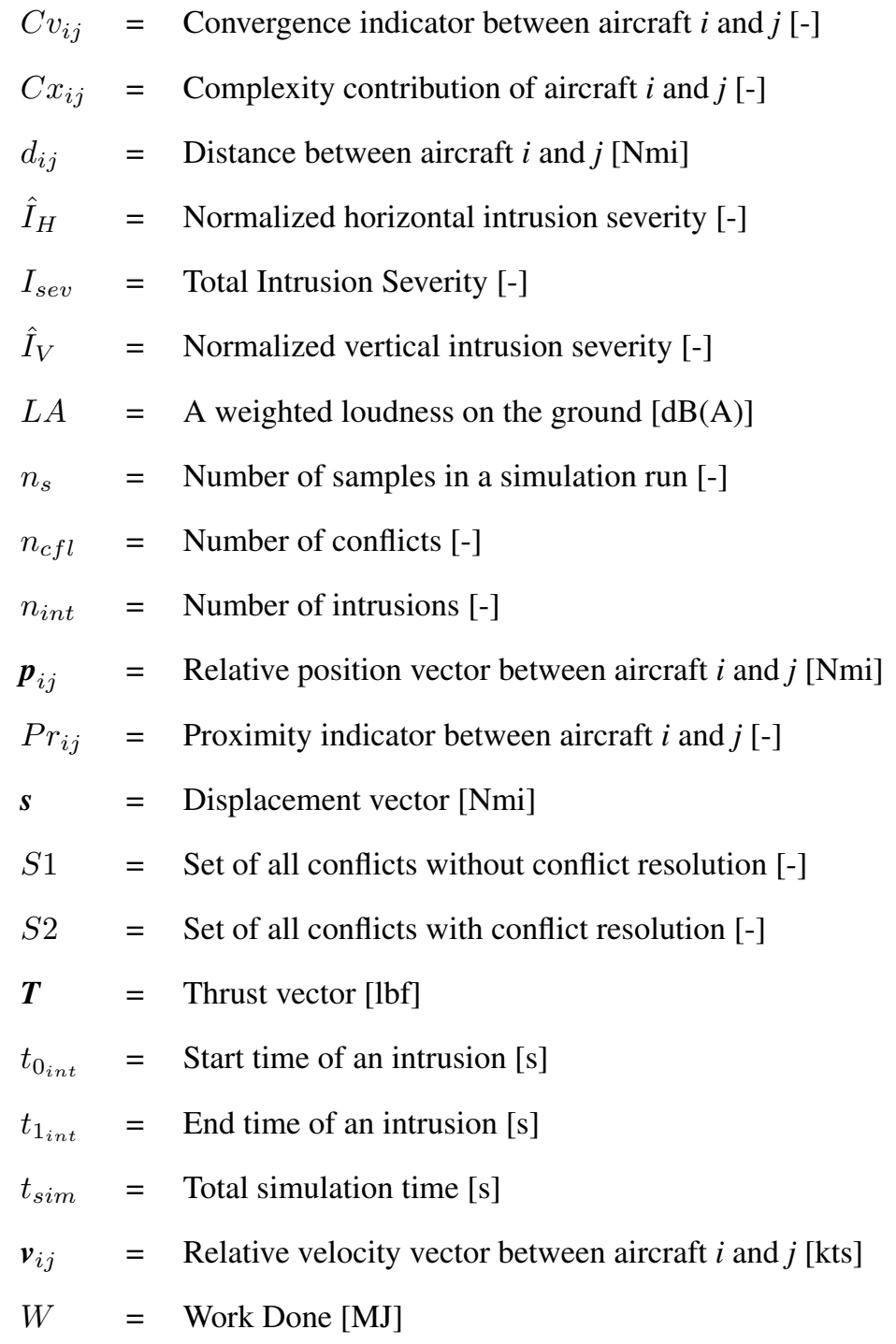

\section{Introduction}

At present, traffic separation in en-route airspace is primarily performed by ground-based Air Traffic Controllers (ATCo), and relies on a rigid network of airways [1,2]. The structure of this network historically depended on the physical location of radio navigation beacons, dating back to the early 1950s [3]. This airway system was devised to help pilots navigate safely under Instrument Flight Rules (IFR), using simple single channel radios [3]. Even though modern airliners have the necessary equipment to fly arbitrary routes accurately, airway navigation is still used during busy daytime operations in most parts of the world [4]. This is because airways limit aircraft flight paths along predefined trajectories, making it easier for the ATCo to monitor and de-conflict traffic flows [5, 6]. However, as airways do not always allow for efficient airspace usage, this centralized airspace design has been widely reported to be nearing saturation levels $[4,7,8,9]$.

In order to cope with the projected increases in traffic demand, a transfer of the traffic separation responsibility 
from the ground to the cockpit has been proposed as an alternative to the current operational model. To realize the resulting decentralized separation paradigm, sometimes referred to as 'self-separation', significant progress has been made towards the development of airborne Conflict Detection and Resolution (CD\&R) algorithms [10]. However, little effort has been devoted towards decentralized airspace design. In particular, there is no consensus in existing literature if some form of traffic organization, or structuring, is also needed to maximize capacity for decentralized separation; while Free Flight researchers advocate that higher densities can be achieved through a reduction of traffic flow constraints $[6,11,12]$, other studies argue that capacity would benefit more from a further structuring of airspace $[13,14,15]$. These diametrically opposed views indicate that the relationship between airspace structure and capacity is not well understood for decentralization, i.e. does more or less structuring lead to a higher capacity? Or, does the degree of structuring required to maximize capacity vary with traffic density?

To answer these questions, this study analyzes the degree of structuring needed to maximize capacity for decentralized separation using fast-time simulations. To this end, four en-route airspace concepts, ranging from a completely unstructured direct routing concept, to a highly structured tube network using 4D trajectories, are compared within the same simulation environment, for multiple traffic demand scenarios. The effect of structure on capacity is subsequently analyzed from the effect of traffic demand variations on safety, efficiency, stability, arrival sequencing, complexity and noise pollution metrics. By including rogue aircraft that ignore concept dependent routing requirements in selected simulation runs, the robustness of the concepts to non-nominal conditions is also analyzed in this study.

The analysis described in this work is performed within the context of a hypothetical Personal Aerial Transportation System (PATS). This setting was adopted to provide the extreme traffic densities, up to 30,000 aircraft per 10,000 square nautical miles, needed to 'stress-test' and compare the four concepts in terms of capacity using a fast-time simulation approach. Note that the aim of this study is not to provide any specific conclusions regarding design options for a future PATS; the focus is only on the analysis of the airspace structure-capacity relationship for decentralized separation.

This paper is structured as follows. Section II defines the notion of airspace structure, and describes the design of four decentralized airspace concepts of increasing structure. This is followed in Section III with the setup of two separate simulation studies that are used to compare the concepts. The results of these simulations are presented and discussed in Sections IV and V, respectively. Finally the main conclusions are summarized in Section VI.

\section{Design of Airspace Concepts}

Airspace structure can be defined as a procedural mechanism for a priori separation and organization of enroute traffic. An example of this in current-day operations is the hemispheric rule, which separates east-bound from west-bound traffic at alternating vertical flight levels [16]. More generally, any a priori structuring of traffic implies posing constraints on one or more of the four degrees of freedom that describe aircraft motion (both spatial as well as temporal). Using this definition of airspace structure, four decentralized airspace concepts, named Full Mix, Layers, 
Zones and Tubes, have been designed by incrementally increasing the number of constraints applied. This section describes and compares the conceptual design of these four airspace concepts.

\section{A. Full Mix}

The Full Mix airspace concept can be most aptly described as 'completely unstructured airspace'. In this concept traffic is subjected to only physical constraints, such as weather, static obstacles and terrain. The Full Mix concept assumes that any structuring of traffic decreases overall system efficiency, and that safety is actually improved by dispersing the traffic over the available airspace. Therefore, aircraft in the Full Mix concept make use of direct horizontal routes, as well as optimum altitudes and velocities, to minimize fuel usage and other related trip costs.

As no level of airspace structure is used to separate potentially conflicting trajectories in Full Mix, safe separation between aircraft is entirely dependent on airborne self-separation automation, see Section III.A.3. Since Full Mix imposes no constraints on the path of aircraft, combined heading, speed and altitude conflict resolution maneuvers are used to reduce deviations from the optimal route.

\section{B. Layers}

The Layers concept can be seen as an extension to the hemispheric rule [16]. In this concept, the airspace is segmented into vertically stacked bands, with each altitude layer limiting horizontal travel to within an allowed heading range. This segmentation of airspace is expected to improve safety when compared to the Full Mix concept, by reducing the probability of conflicts with crossing traffic for cruising aircraft. However, this increased safety comes at the price of efficiency; while direct horizontal routes are still possible, vertical flight profiles are dictated by the bearing between origin and destination, and the corresponding altitude band with the required heading range. As a result, flights may not be able to cruise at their optimum flight levels, increasing fuel burn when compared to Full Mix. An exception to the heading-altitude rule is made for climbing and descending aircraft; these aircraft are allowed to maintain heading

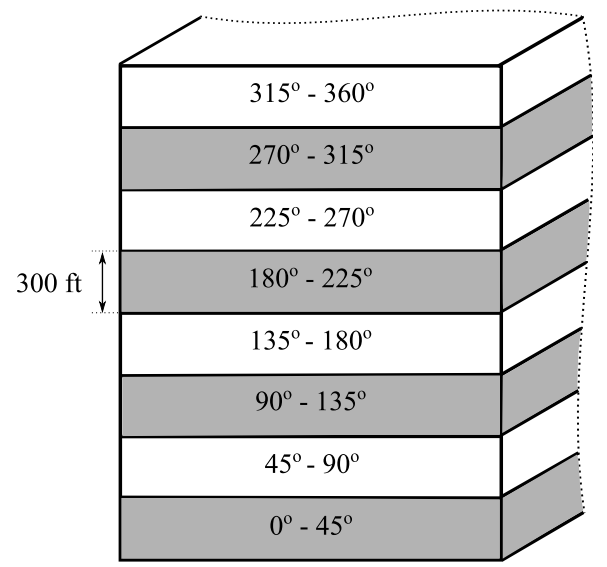

Figure 1. Isometric view of the Layers concept with one complete set of altitude bands. Here, each altitude band constrains aircraft headings to within a predefined range. 
while climbing or descending to their destination altitude.

Figure 1 displays a schematic of the Layers concept. Here it can be seen that each altitude layer corresponds to a heading range of $45^{\circ}$ and has a height of $300 \mathrm{ft}$. With these dimensions, two complete sets of layers fit within the airspace volume used to compare concepts, see Section III.B.1. As a result, short flights can stay at low altitudes while longer flights can improve fuel burn by flying at higher flight levels. This is expected to mitigate the efficiency drop of predetermined altitudes in this concept.

The Layers concept also makes use of the same self-separation automation utilized by Full Mix, albeit with restrictions on the allowed resolution maneuvers. While combined heading, speed and altitude resolutions are permitted for climbing and descending traffic, for cruising aircraft, altitude resolutions would create new conflicts with traffic in adjacent layers. Resolutions are therefore limited to combined heading and speed maneuvers for cruising aircraft.

\section{Zones}

Similar to Layers, the Zones concept separates traffic based on similarity of travel direction. However, in this case, a horizontal segmentation of airspace is used to separate traffic along pre-defined trajectories. In this respect, the Zones concept resembles the airway-based airspace design used today in that it facilitates travel towards and away from locations with high traffic demand.

The horizontal topology used by the Zones concept, see in Figure 2, consists of two major zone types: radial and ring zones. Radial zones separate inbound and outbound traffic from the center of the topology, which coincides with an area with high traffic demand. Concentric ring zones, on the other hand, function as connections between the radial zones, and separates clockwise and anti-clockwise traffic flows from each other. Aircraft can travel between any two points in the topology using a combination of radial and ring zones. As there is no vertical segmentation of airspace in this concept, optimum altitudes are selected based on the planned flight distance between origin and destination. The Zones concept used for simulations consisted of 41 rings. The number of radials depended on the distance from the center of the topology to ensure adequate separation between adjacent radials; 72 radials were used for distances up to

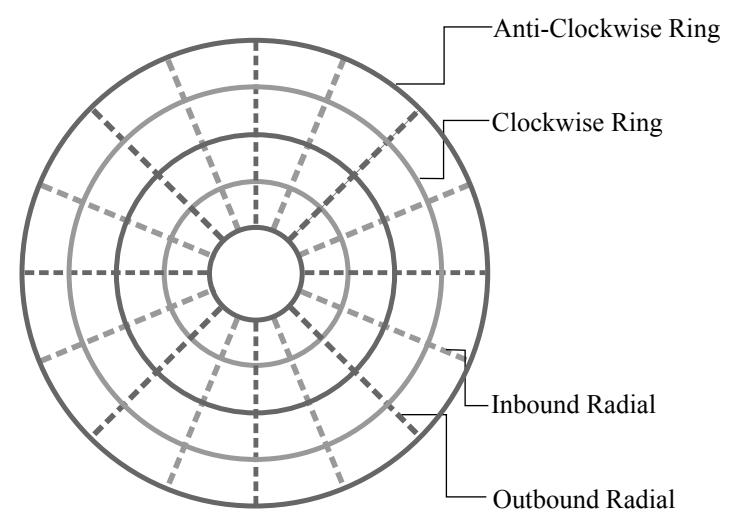

Figure 2. Top down view of an example Zones concept, which constrains traffic in the horizontal plane. 
$6 \mathrm{Nmi}$ from the center, after which 216 radials were defined.

The Zones concept also uses self-separation automation to separate aircraft flying within the same zone, as well as to assist with the merging of aircraft between ring and radial zones. Since the Zones topology dictates the horizontal path of an aircraft, heading resolutions are not allowed for this concept.

\section{Tubes}

As a maximum structuring of airspace, the final concept implements four-dimensional tubes that provide a fixed route structure in the air. Here, the aim is to increase the predictability of traffic flows by using preplanned conflict free routes.

The tube topology can be thought of as a graph with nodes and edges, see Figure 3. The nodes are connection points for one or more routes, while the edges are the tubes connecting two nodes. Tubes at the same horizontal level never intersect, except at the nodes, and are dimensioned to fit exactly one aircraft in the vertical and horizontal plane. To provide multiple route alternatives, the Tubes implementation uses a total of 13 tube layers that are placed above each other, with decreasing granularity. This way, short flights profit from a fine grid at the lowest layer, while at the same time, longer flights benefit from lengthier tubes at higher layers. Finally, it should be noted that aircraft are only allowed to climb/descend through one tube layer at a time.

Unlike the other concepts, the Tubes concept uses time-based separation. This mode of separation dictates that when an aircraft passes a node, it will 'occupy' that node for a prescribed time interval. Within this occupancy interval no other aircraft is allowed to pass through that node to prevent conflicts. For each node, an interval list is maintained that keeps track of the times at which that node is expected to be occupied. These lists are shared between all aircraft, and updated whenever new flights enter the network. Furthermore, new flights are only allowed to select routes that are not predicted to conflict with existing aircraft in the network. To ensure that separation at the nodes also guarantees separation within the tubes, all aircraft within the same layer are required to fly at the same velocity. This prescribed speed increases with the altitude of the layer to match the decreasing granularity of the tube network. A major advantage of this mode of separation is that it allows the tube network to be bidirectional, as the occupancy

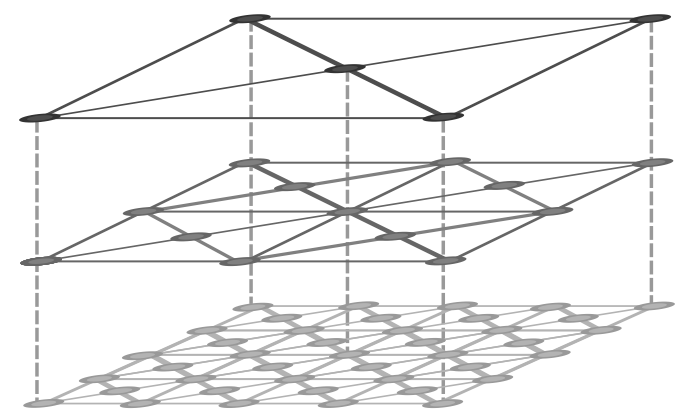

Figure 3. Isometric view of an example Tubes topology, which constrains traffic along all four dimensions of motion. The dashed lines are used to indicate the placement of nodes above each other. 


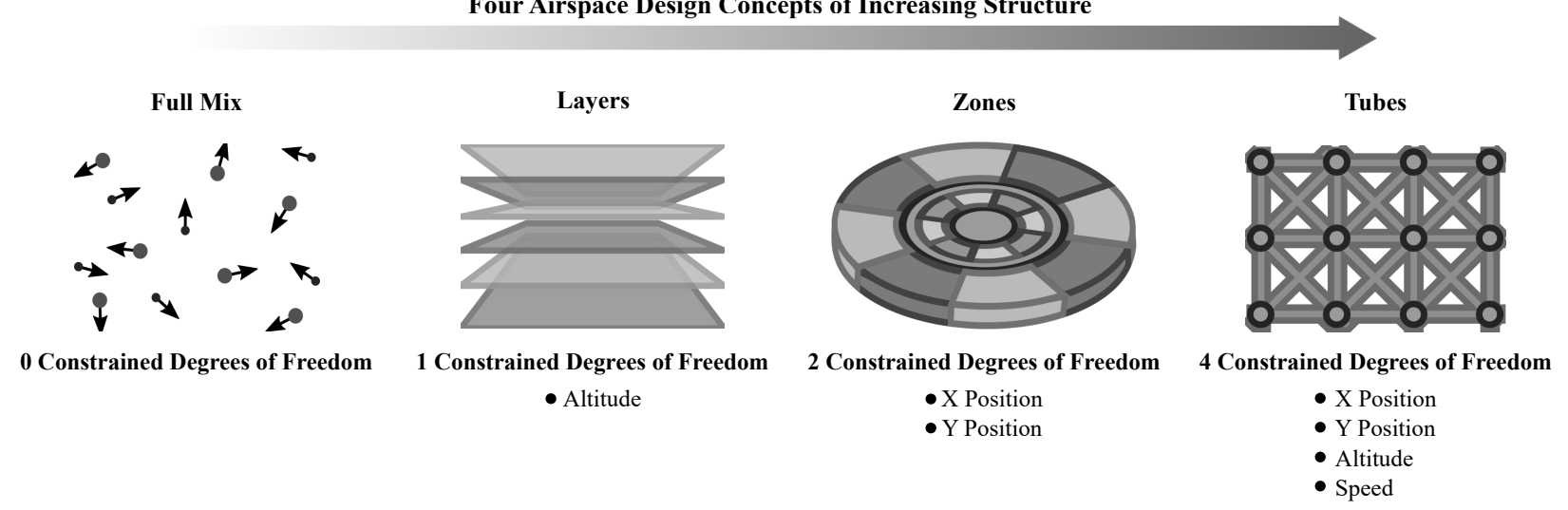

Figure 4. Four point airspace structuring framework. The level of structure is increased from Full Mix to Tubes by incrementally increasing the number of constraints applied.

of a node is independent of travel direction. This simplifies its design, and enables a closer packing of tubes in the topology.

The time-based separation and preplanned routes used by the Tubes concept makes it similar to some 4D Trajectory Based Operations (TBO) concepts that have been discussed in literature. However, it should be noted that most TBO concepts utilize a centralized planning approach in which globally optimum routes are determined using an iterative approach, after negotiations between an airspace user and an Air Navigation Service Provider (ANSP) [13]. Aircraft in the Tubes concept, on the other hand, use decentralized route planning whereby the shortest (conflict free) route is selected, given the availability of the tube topology at the time of flight plan calculation, i.e., routes are selected in Tubes using the 'first-come-first-served' principle.

\section{E. Concept Comparison}

Figure 4 compares the four airspace concepts described above in terms of the number of constrained degrees of freedom. The figure shows that options for trajectory planning become increasingly restricted as the degree of structure is incrementally increased from Full Mix to Tubes; while all four degrees of freedom are available in Full Mix, in the Tubes concept, aircraft have no degrees of freedom, and are required to rigidly follow preplanned space-time routes through a predefined topology. Between these two extremes, the Layers and Zones concepts were defined to allow three and two degrees of freedom respectively. This choice was made such that the four concepts span the entire range from unstructured to structured airspace. Thus, a comparison of the four concepts using fast-time simulations can be used to analyze the structure-capacity relationship for decentralized airspace. The goal of these simulations is not to arrive at precise capacity estimations for the four concepts, but rather to consider on how the degree of structuring affects capacity. Correspondingly, the concepts are subjected to multiple traffic demand densities, and a relative capacity ranking is performed by measuring the effect of traffic density changes on several airspace metrics. 


\section{Simulation Design}

Two separate fast-time simulation experiments were conducted to compare the four airspace concepts in terms of capacity and robustness. This section describes the design of these two experiments.

\section{A. Simulation Development}

\section{Simulation Platform and Vehicle Modeling}

The Traffic Manager (TMX) software, developed by the National Aerospace Laboratory of the Netherlands (NLR), was used as the simulation platform in this research. The TMX simulator is well established in the ATM research community, and has been used in many previous Air Traffic Management (ATM) related simulation studies. For more information on TMX capabilities, the reader is referred to [17].

In order to simulate Personal Aerial Vehicle (PAV) dynamics, parameters of existing point-mass aircraft models in TMX, which are based on Eurocontrol's BADA Aircraft Performance Models (APM) [18], were adapted to match the performance specifications of several PAVs that are currently under development. In total, three different PAV types were used in the simulations.

\section{Concept Implementation}

The four concepts were implemented by modifying TMX's trajectory planning functions. The Full Mix concept used the direct horizontal route and the most fuel efficient altitude, as determined by the APMs. Layers also used the direct horizontal trajectory. However, altitude was selected based on the bearing to the destination and the matching altitude from a predefined list (see Section II.B). Additionally, distance determined the choice between the upper and lower layer sets; flights with a cruising distance less than $22 \mathrm{Nmi}$ used the lower layer set.

For the Zones concept, the $\mathrm{A}^{*}$ path planning algorithm was used to determine the shortest route over its predefined horizontal topology, while the most fuel efficient altitude was chosen by the APMs. Tubes also employed A* to calculate the shortest path, but in this case, it was also used to examine whether the selected route was conflict-free. Here, an instantaneous planning approach was used whereby the occupancy of each node along a proposed route was checked at traffic desired departure times. If any node along a proposed route was found to be occupied by another flight, the corresponding route was discarded, and the $\mathrm{A}^{*}$ algorithm backtracked to evaluate the next best solution. If no route could be found, a pre-departure delay was applied in multiples of 10 seconds up to a maximum of 30 minutes. After this period, the tube network was considered to be saturated, and that flight was canceled. Once an appropriate route was found, aircraft were required to follow their preplanned routes as closely as possible, including arrival time intervals at each node.

For both Zones and Tubes, the $\mathrm{A}^{*}$ algorithm used in this work had to be modified from the generic version. This was because origins and destinations for traffic were located outside the airspace volume used to design the concepts 
(see Section III.B.1). Consequently, the generic $\mathrm{A}^{*}$ algorithm could yield unfeasible solutions due to aircraft bank angle limitations imposed by the APMs. To solve this problem, the A* implementation in this work differs slightly from the conventional algorithm by allowing already visited nodes to be rechecked if bank angle limits were exceeded, i.e., by remarking 'closed nodes' as 'open nodes'. A complete description of the A* algorithm can be found in [19].

\section{Self-Separation Automation}

The Full Mix, Layers and Zones concepts relied on airborne self-separation automation for tactical separation. It consisted of separate Conflict Detection (CD), Conflict Resolution (CR) and Conflict Prevention (CP) modules. CD was performed through linear extrapolation of aircraft positions over a prescribed 'look-ahead' time. Once conflicts were predicted, the Modified Voltage Potential (MVP) algorithm was used for CR in a pair wise fashion, resulting in implicit cooperative resolution strategies. Finally, the CP algorithm ensured that aircraft did not turn into conflicts, in an effort to mitigate conflict chain reactions. Previous research showed that this three pronged system was highly effective in solving multi-aircraft conflicts. Furthermore, this system was found to be computationally efficient as each aircraft is only concerned with its own separation with neighboring traffic. For more details, please consult [20].

Based on initial test runs, a look-ahead time of 60 seconds, as well as separation margins of 0.135 Nmi horizontally and $150 \mathrm{ft}$ vertically, were found to be suitable for the APMs used here. Since the focus of this study is on decentralized en-route airspace, self-separation was performed only within the experiment airspace block, defined to be between 1650-6500 ft (see Section III.B.1). Additionally, aircraft were assumed to have perfect knowledge of the states of neighboring traffic to focus exclusively on the structure-capacity relationship.

\section{Wind}

In real-life operations, wind uncertainties are a common source of prediction error. To take this effect into account, wind was deliberately omitted from the simulation's trajectory planning functions to study the effect of uncertainties, which could cause deviations from the planned trajectory, on the four airspace concepts. To this end, wind was modeled as a uniform and time-invariant vector field with random direction and random speed (12-22 kts). Although these conditions cannot be assumed by default, for a short time interval in a small observed area, and at sufficient altitudes to clear ground obstacles, as for the current study, this assumption is adequate for the purposes of this work.

\section{B. Traffic Scenarios}

\section{Testing Region and Flight Profiles}

To create high density traffic scenarios, a small square region, with an area of $1600 \mathrm{Nmi}^{2}$, was used for traffic simulations. Although this research focuses on en-route airspace design, arrival and departure operations were simulated to ensure that the results did not ignore the impact of ground constraints. Correspondingly, aircraft took-off from one of $1600 \mathrm{arrival} / \mathrm{departure}$ locations that were evenly distributed at ground level, against the direction of the wind. To 
prevent take-off conflicts, aircraft had a minimum creation interval of 60 seconds at each origin, corresponding to the CD look-ahead time.

After take-off, aircraft climbed to the 'experiment airspace block', defined between $1650 \mathrm{ft}$ and $6500 \mathrm{ft}$. Once aircraft entered the experiment block, they followed concept dependent routing requirements, both horizontally and vertically. At a predetermined distance from their destination (which depended on the cruise altitude), aircraft descended out of the experiment block. Standardized climb/descent profiles were used below the experiment block for all airspace concepts, and were specified using the APMs. To simplify simulation development, runway capacity was not managed during landing. Instead, the effect of arrivals was analyzed using the 'arrival sequencing' metric, see Section III.E.4.

Relatively low altitudes were selected for the experiment block to limit the horizontal area needed for simulation. Given the constant climb/descent profiles below the experiment block, higher altitudes would have increased the horizontal area needed for the simulations. A larger area would in turn increase the traffic volumes needed to realize the desired densities, increasing computational effort without tangible benefits in terms of the primary research goals. On a similar note, all aircraft took-off from and landed at one of the 1600 arrival/departure locations that were defined in the square simulation area. Interactions with aircraft outside this area would not contribute heavily to an understanding of the airspace structure-capacity relationship, particularly for decentralization. Thus, such interactions are outside the scope of this work.

\section{Traffic Demand}

Four traffic demand scenarios of increasing density were used to compare the concepts, and were defined in terms of the instantaneous number of aircraft in the air, see Table 1. These scenarios had a an average trip distance and speed of $30 \mathrm{Nmi}$ and $120 \mathrm{kts}$, respectively, and made use of assumptions for future per capita demand for PAVs, see [21] for more details.

Table 1. Instantaneous Traffic Volume of the Four Demand Scenarios

\begin{tabular}{c|cccc} 
Scenario & Low & Medium & High & Ultra \\
\hline $\begin{array}{c}\text { Instantaneous Traffic } \\
\text { Volume }\end{array}$ & 2,625 & 3,375 & 4,125 & 4,875
\end{tabular}

In addition to different demand volumes, traffic scenarios were created with different demand patterns. Here, scenarios with largely converging, diverging and 'mixed' demand patterns were used. These different demand patterns were created by varying the ratio of origins and destinations that acted as sources and sinks, and by varying the aircraft creation time intervals in different regions of the simulation area. 


\section{Rogue Aircraft}

In a separate experiment, 'rogue aircraft' were introduced at random time intervals. Their separation requirements were seven times larger horizontally, and four times larger vertically, when compared to normal aircraft in the simulation. These aircraft flew haphazardly through the airspace with continuously varying heading and altitude. Furthermore, rogue aircraft were non-cooperative. This meant that normal aircraft were solely responsible for detecting and resolving conflicts with rogue aircraft using its self-separation automation, in all concepts. Although time based separation is used in Tubes, the self-separation automation described above is used with speed resolutions to resolve conflicts with rogue aircraft alone. By monitoring the effect of rogue aircraft on safety metrics, the robustness of the four concepts to non-nominal events can be analyzed.

\section{Simulation Procedure and Data Logging}

To enable a fair comparison between all concepts, standardized simulation conditions were used to minimize unsystematic variation in the results. For a particular repetition of a traffic demand volume, the creation times of aircraft, the origin-destination combinations, the strength and direction of the simulated wind, as well as the introduction and trajectories of rogue aircraft (in non-nominal experiment), were kept constant across all concepts. Additionally, scenarios had a duration of two hours, consisting of a 45 minute (traffic volume) build up period, a 1 hour logging period, during which the traffic volume was held constant at the required level, and a 15 minute wind down period, required to allow aircraft created during the logging hour to finish their flights, and prevent abnormally short flights from skewing the results.

Two types of logging were used. Event-driven logging kept track of the properties of conflicts and intrusions as they occurred. It was also used to store flight efficiency data when an aircraft arrived at its destination, including the time interval between consecutive arrivals at each destination. Periodic logging was used to monitor the status of all flights in the experiment airspace block every 30 seconds. This was required to monitor traffic volumes, as well as for computing structural complexity and noise pollution metrics.

\section{Independent Variables}

Two separate experiments were performed: the nominal experiment and the non-nominal experiment.

\section{Nominal Experiment}

The nominal experiment focused on the impact of airspace structure on capacity under ideal conditions; although traffic was subjected to a uniform wind field (see Section III.A.4), no other detriments to aircraft motion were included in this experiment. The two independent variables of this experiment, and their levels, are:

1. Airspace concept: Full Mix, Layers, Zones and Tubes 
2. Traffic demand: Low, Medium, High and Ultra (see Table 1)

For each of the 16 experiment conditions, six repetitions were performed, consisting of two converging, two diverging and two mixed traffic demand patterns. Additionally, the scenarios were also simulated with and without tactical CR to measure airspace stability, resulting in a total of 192 nominal simulation runs (4 concepts $\mathrm{x} 4 \mathrm{demand}$ scenarios x 6 repetitions $\mathrm{x} 2$ CR settings).

\section{Non-Nominal Experiment}

The goal of the second experiment was to compare the relative robustness of the concepts to non-nominal situations. For this purpose, rogue aircraft were added to traffic scenarios during the logging hour, in addition to wind. The two independent variables of this experiment, and their levels, are:

\section{Airspace concept: Full Mix, Layers, Zones and Tubes}

2. Number of rogue aircraft: $4,8,16$ and 32

The resulting 16 non-nominal experiment conditions were performed using the 'Medium' traffic demand scenario listed in Table 1. Once again, six repetitions, as well as simulations with and without tactical CR were performed, resulting in a total of 192 non-nominal simulation runs (4 concepts x 4 rogue scenarios x 6 repetitions x 2 CR settings).

\section{E. Dependent Variables}

Six categories of dependent variables are used to compare the concepts: safety, efficiency, stability, arrival sequencing, structural complexity and noise pollution. The metrics used to access each category are described below.

\section{Safety}

Safety metrics focus on the ability of an airspace concept to maintain safe separation between aircraft. Separation performance is measured in terms of the number of intrusions and conflicts. Here, intrusions are defined as violations of minimum separation requirements, while conflicts are defined as predicted intrusions, i.e., when two (or more) aircraft are expected to violate separation requirements within a predetermined 'look-ahead' time (60 seconds in this research).

Intrusions do not imply collisions. Therefore, in addition to counting the number of intrusions, it is important to consider the severity of an intrusion. The severity of an intrusion, $I_{\text {sev }}$, is dependent on the path of an aircraft through the protected zone of another, see Figure 5, and is computed using the following expression:

$$
I_{\text {sev }}=\max _{t_{0_{\text {int }}}-t_{1_{\text {int }}}}\left[\min \left(\hat{I}_{H}(t), \hat{I}_{V}(t)\right)\right]
$$




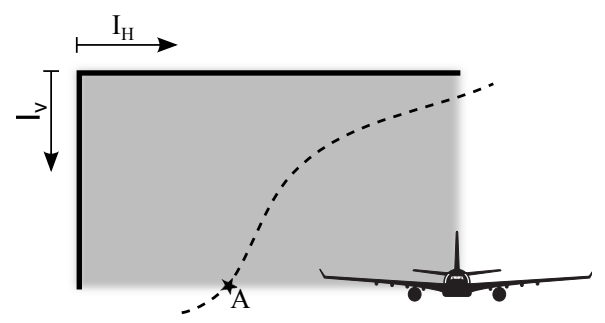

Figure 5. Front view of an intrusion. The dashed line shows the intrusion path of an aircraft through the protected zone of another.

Here, $\hat{I}_{H}$ and $\hat{I}_{V}$ are the horizontal and vertical intrusions that are normalized with respect to the corresponding minimum separation requirements, while $t_{0_{\text {int }}}$ and $t_{1_{\text {int }}}$ are the start and end times of an intrusion. Using the above relation, the intrusion severity for the intrusion path shown in Figure 5 is equal to the normalized horizontal intrusion at point 'A'.

The last safety metric is Intrusion Prevention Rate (IPR). As the name suggests, this metric considers the proportion of intrusions that were successfully avoided, and is computed as follows:

$$
I P R=\frac{n_{c f l}-n_{\text {int }}}{n_{c f l}}
$$

Here, $n_{c f l}$ and $n_{\text {int }}$ are the number of conflicts and intrusions, respectively.

\section{Efficiency}

The efficiency of the concepts is analyzed using the work done metric. This metric considers the optimality of an aircraft's trajectory, and therefore has a strong correlation with fuel/energy consumption. For each flight, the work done, $W$, is computed as:

$$
W=\int_{\text {path }} \mathbf{T} \cdot d \mathbf{s}
$$

Here, $\mathbf{T}$ is the thrust vector and $\mathbf{s}$ is the displacement vector.

\section{Stability}

Resolving conflicts may cause new conflicts at very high traffic densities due to the scarcity of airspace. The stability of the airspace as a direct result of conflict resolution maneuvers has been measured in literature using the Domino Effect Parameter (DEP) [12, 22]. The DEP can be visualized through the Venn diagram pictured in Figure 6. Here, $\mathrm{S} 1$ is the set of all conflicts without resolutions, and S2 is the set of all conflicts with resolutions, for identical traffic scenarios. Furthermore, three regions can be identified in Figure 6 from the union and relative complements of the two sets, with $R 1=S 1 \backslash S 2, R 2=S 1 \cup S 2$ and $R 3=S 2 \backslash S 1$. 


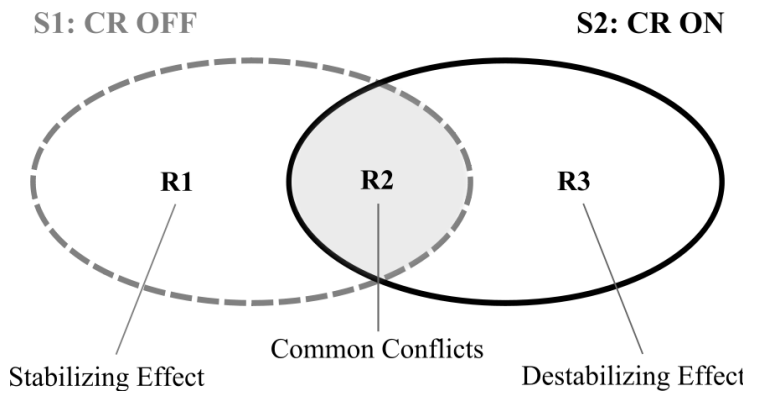

Figure 6. The Domino Effect Parameter (DEP) compares simulations with and without Conflict Resolution (CR) to measure airspace stability

By comparing the number of R1 and R3 conflicts, the proportion of additional 'destabilizing' conflicts that were triggered by resolution maneuvers can be determined. Thus, the DEP is defined as [22]:

$$
D E P=\frac{R 3-R 1}{S 1}=\frac{S 2}{S 1}-1
$$

It should be noted that the Tubes concept does not use tactical conflict resolution (expect with rogue aircraft). Hence, the DEP has no meaning for Tubes.

\section{Arrival Sequencing}

Although runway occupancy was not managed for landing traffic, the effect of airspace structure on approach sequencing is examined a posteriori by considering the time interval between successive arrivals at each destination. Both the number of arrivals within 60 seconds each other (equaling the CD look-ahead time), and the average time interval between successive arrivals, at each destination, has been used to measure arrival sequencing.

\section{Structural Complexity}

Complexity metrics are used to measure the difficulty of controlling a given traffic situation. Although complexity in ATM research often refers to ATCo workload, the class of intrinsic, or geographical, complexity metrics, which only consider the traffic patterns generated by an airspace concept, is most appropriate for this research.

Two indicators are used to measure intrinsic complexity. The proximity indicator, $\mathrm{Pr}$, describes the geographical distribution of aircraft within a specified volume of airspace, enabling the identification of spatial zones with high levels of aggregation, relative to the considered volume. On the other hand, the convergence indicator, $C v$, measures the geometric distribution of aircraft speed vectors to distinguish between converging and diverging traffic flows [23].

To compute intrinsic complexity, for each aircraft under consideration, a spatial weighting window that is centered on that aircraft is opened. Then, a complexity metric associated with the reference aircraft is determined by adding together the product of the two above complexity factors for all pairs of aircraft within the reference window: 


$$
C x_{i}=\lambda \sum_{j / C v_{i j} \leq 0} \overbrace{-\frac{\mathbf{p}_{i j} \cdot \mathbf{v}_{i j}}{d_{i j}} \cdot \underbrace{d_{i j}} \cdot \underbrace{e^{-\alpha d_{i j}^{2}}}_{P r_{i j}}}^{\underbrace{}_{P r_{i j}}}
$$

Here, subscripts $i$ and $j$ represent the two aircraft considered, $\alpha$ and $\lambda$ are parameters fixed by the user, $d_{i j}$ is the normalized distance between aircraft, and $\mathbf{p}_{i j}$ and $\mathbf{v}_{i j}$ represent the relative position and speed vectors, respectively.

Although aircraft positions are known accurately within the simulation, in real life operations, there is always some uncertainty regarding the precise location of aircraft. To ensure a reliable and robust complexity analysis, it is necessary to take this effect into account. Therefore, the complexity metric shown above is extended by considering all possible pairs of trajectory samples within a spatio-temporal window that is centered on each aircraft, i.e., by time averaging the complexity metric $C x_{i j}$ from eq. 5 over all pairs of samples $i j$ within a specified time interval. Finally, the robust complexity for a given traffic situation is calculated as the sum of the robust complexity metrics for all aircraft present in the airspace under evaluation.

Since complexity is defined for a particular traffic situation at a given time instant, for easier comparison of concepts, it is necessary to aggregate the robust complexity metric, $C x(t)$, over the total number of samples taken for each simulation run, $n_{s}$. This is done using a structural complexity metric, $S C x$, defined as:

$$
S C x=\frac{1}{n^{*}} \sum_{t=0}^{n_{s}} C x(t)+\beta \max _{t} C x(t) ; n^{*}=\max _{s} n_{s}
$$

Here, $\beta$ is a parameter fixed by the user to represent the relative importance of the maximum complexity compared to the average complexity, for a particular simulation run. In this work, $\beta=0.05$ was selected. It should be noted that no differences to the trend of the $S C x$ metric were found for $\beta$ values between 0.01 and 0.3 . For more details on the complexity metrics used, please refer to [24].

\section{Noise Pollution}

Noise pollution is analyzed using the $L A_{e q}$ noise metric, calculated as [25]:

$$
L A_{e q}=10 \log _{10}\left(\frac{1}{t_{\text {sim }}} \int_{0}^{t_{\text {sim }}} 10^{\frac{L A(t)}{10}} d t\right)
$$

where $t_{\text {sim }}$ is equal to one hour, corresponding to the duration of the logging hour, and $L A(t)$ is the time dependent, A-weighted loudness level on the ground due to all the aircraft in a scenario, in $\mathrm{dB}(\mathrm{A})$. The computation of $L A(t)$ uses noise-power-distance relations that are similar to those found in environmental noise prediction programs such as the Integrated Noise Model (INM) ) [26]. These relations (not shown) were established based on the data that is available for PAVs currently being designed, including the Maximum Take-Off Weight (MTOW) and FAA regulations 
governing the maximum noise that is allowed to be generated by tilt-rotor aircraft.

The footprints of the $L A_{e q}$ metric are assessed by calculating iso-contour lines at the $68 \mathrm{~dB}(\mathrm{~A})$ level. Although noise regulations in the Netherlands are based on the 48 and $58 \mathrm{~dB}(\mathrm{~A})$ Loudness Day Evening Night (LDEN) sound levels, no requirements have been specified for the large amount of PAV traffic considered in this work. Hence, the next level, $68 \mathrm{~dB}(\mathrm{~A})$, was selected for the current analysis. The encapsulated area of this contour can be used to compare concepts. A larger contour area implies a larger exposure to noise pollution. More details on the noise modeling method used in this work can be found in [27].

\section{Results}

In this section, the results of the nominal and non-nominal experiments are presented separately. For both experiments, the effect of the independent variables (airspace concept and traffic demand/number of rogue aircraft) on the dependent measures is analyzed using error bar charts, displaying the mean and the $95 \%$ confidence interval for each simulation condition. As identical scenarios were performed with and without tactical CR, whenever relevant, separate error bar charts are used to assess the need and the effect of CR on the four concepts. These charts are created by consolidating the demand data per concept.

\section{A. Nominal Experiment}

More than six million individual flights were simulated during the nominal experiment. Of these, data from approximately three million flights that flew during the logging hour have been analyzed. To gain a better sense on the amount of traffic simulated, as well as to explain the consequent implications on the analysis of the dependent variables, it is first necessary to consider the traffic volumes and densities that were actually realized during the experiment.

\section{Traffic Volume and Density}

Figure 7 a shows the total traffic volume simulated during the logging hour, per simulation run, for all concept-demand combinations. Here it can be seen that the traffic volume simulated for the Full Mix, Layers and Zones concepts

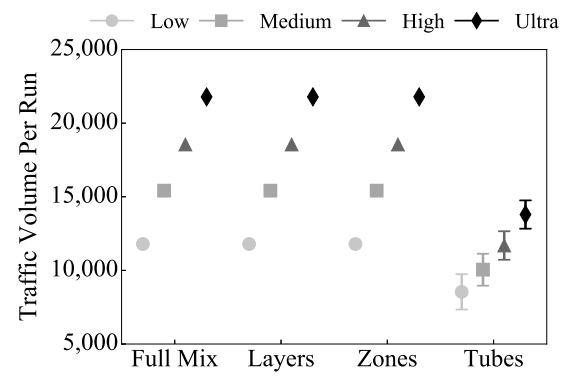

a) Total traffic volume

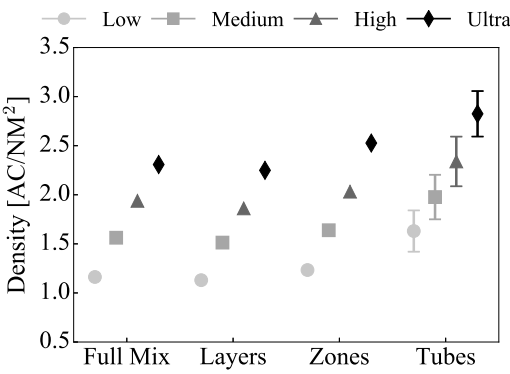

b) Average traffic density

Figure 7. Means and $95 \%$ confidence intervals of the total number of flights and the average density per simulation run 
are the same and range from 12,000 aircraft in the Low scenario, to 22,000 aircraft in the Ultra scenario. On the other hand, the Tubes concept is shown to deviate from the other concepts for all demand conditions, even though all concepts were subjected to the same scenarios. In fact, the maximum traffic volume achieved by the Tubes concept in the Ultra scenario is less than the Medium demand volume realized by the three less structured concepts, indicating that demand could not be met by Tubes. This is because the Tubes concept used pre-departure delays and flight cancellations whenever conflict free routes could not be found at scenario specified departure times. As other concepts did not have the ability to delay or cancel flights, all flights in the demand scenarios were simulated.

In addition to total volume, it is also necessary to consider the traffic densities realized during the simulation, see Figure 7b. Here it is clear that traffic density is fairly similar for the Full Mix, Layers and Zones concepts for all demand conditions. Conversely, the Tubes concept resulted in the highest traffic densities, despite the lower number of flights simulated for all demand scenarios (compare Figure 7a with Figure 7b). This paradoxical result can be explained by the fact that aircraft in the Tubes concept were often forced to use indirect and longer routes when the shortest path between an origin-destination pair was congested i.e., not conflict free prior to departure. Thus, average distances were much higher for Tubes (see efficiency metrics), causing aircraft to exist for longer durations in the experiment volume, which in turn resulted in higher densities than the other concepts.

These differences in traffic volume and density for the Tubes concept need to be taken into account when considering the other dependent variables. Although Figure 7a suggests that Tubes has a lower airspace capacity when compared to the other concepts, it should be noted that the figure does not imply that the other concepts are able to, for instance, facilitate the higher volumes safely. Therefore, conclusions with respect to capacity also depend on the other dependent variables discussed below, and cannot be based purely on the amount of traffic simulated. Moreover, whenever appropriate, these metrics are computed relative to the number of flights simulated to allow for a fair comparison between concepts.

\section{Safety}

The number of conflicts and intrusions per flight for all simulation conditions are displayed in Figures 8 and 9, respectively. As expected, the number of conflicts and intrusions increased with traffic demand for all concepts, see Figures 8a and 9a. Furthermore, the figures also show that the more structured Zones and Tubes concepts led to significantly higher numbers of conflicts and intrusions compared to the less structured Full Mix and Layers concepts.

The effect of tactical CR on the number of conflicts and intrusions is pictured in Figures 8b and 9b, respectively. Here, the results with CR ON reflect the safety of each concept as a whole, whereas the results with CR OFF shows how well concepts are able to prevent conflicts from occurring. As Tubes did not use tactical CR, there were no differences between the $\mathrm{ON}$ and $\mathrm{OFF}$ conditions. For the other three concepts, the number of intrusions was considerably reduced with CR ON. However, the effect of CR on the number of conflicts did not follow the same trend. For Full Mix and Zones, the number of conflicts increased with CR ON. This was expected, as resolution maneuvers increase flight 


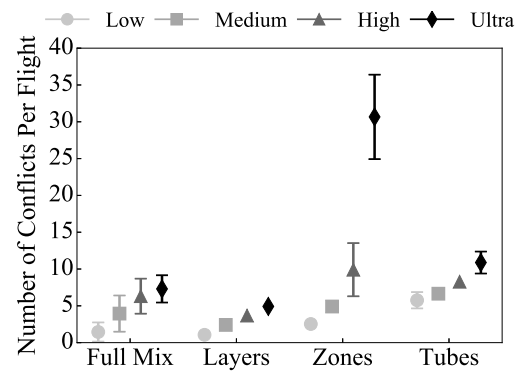

a) Effect of traffic demand (with conflict resolution)

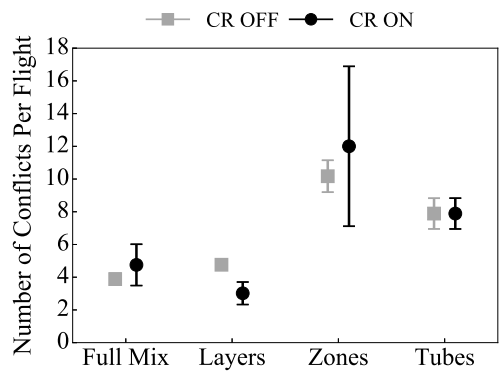

b) Effect of Conflict Resolution (CR)

Figure 8. Means and $95 \%$ confidence intervals of the number of conflicts per flight

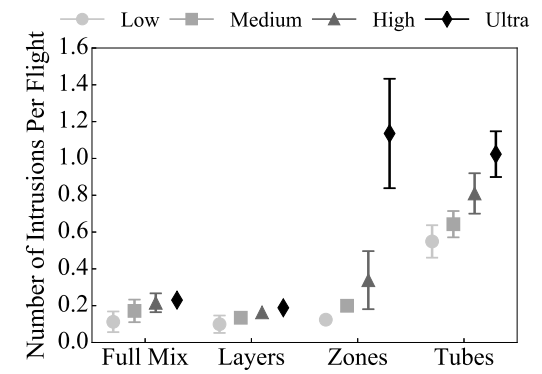

a) Effect of traffic demand (with conflict resolution)

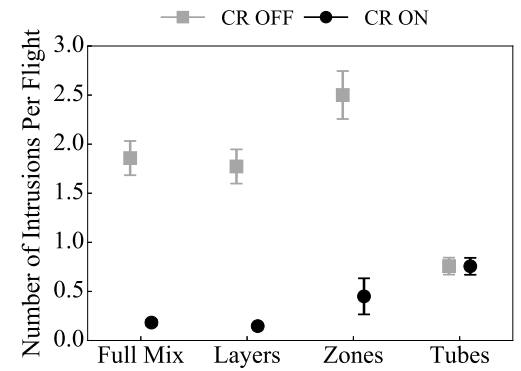

b) Effect of Conflict Resolution (CR)

Figure 9. Means and $95 \%$ confidence intervals of the number of intrusions per flight

distances and the consequent probability of encountering other aircraft. However, for the Layers concept, the opposite was found, with CR ON leading to a lower number of conflicts. This unusual result is further analyzed using stability metrics.

It is also worth noting that the Tubes concept, which aimed at de-conflicting flights prior to take-off, resulted in a very high number of conflicts and intrusions for all scenarios. This was because the trajectory planning functions used in the Tubes concept did not take uncertainties, such as wind, into account. These uncertainties caused aircraft to deviate from their planned flight paths during the simulation, resulting in a large number of conflicts due to the tight packing of the Tubes topology. As no tactical CR was used in the Tubes concept, these conflicts also resulted in a large number of intrusions.

To further analyze trends in the number of safety incidents, the relative velocity magnitudes between conflicting aircraft is computed, see Figure 10. Here, only conflicts between cruising aircraft, and data for simulations with CR OFF, are used, in order to consider the inherent safety of each concept. From this figure, it is clear that the vertical airspace segmentation used by the Layers concept significantly reduced relative velocities compared to the other three concepts, explaining the high safety of this concept.

The effect of the independent variables on intrusion severity is pictured in Figure 11. Here it can be seen that the Zones concept resulted in the lowest intrusion severity, despite experiencing the highest number of conflicts and 


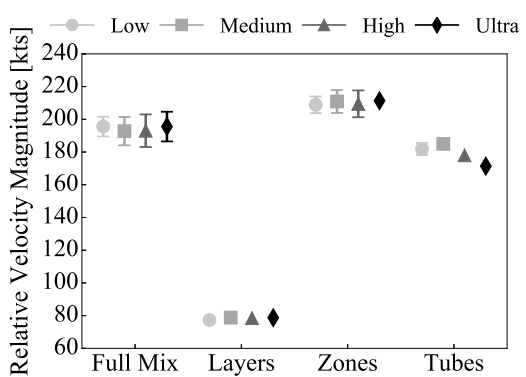

Figure 10. Means and $95 \%$ confidence intervals of the relative velocity magnitudes between conflicting aircraft in cruise (without conflict resolution)

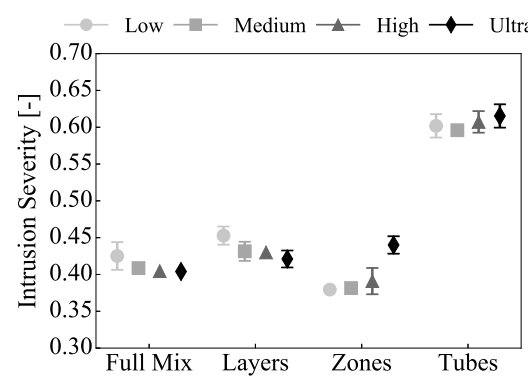

a) Effect of traffic demand (with conflict resolution)

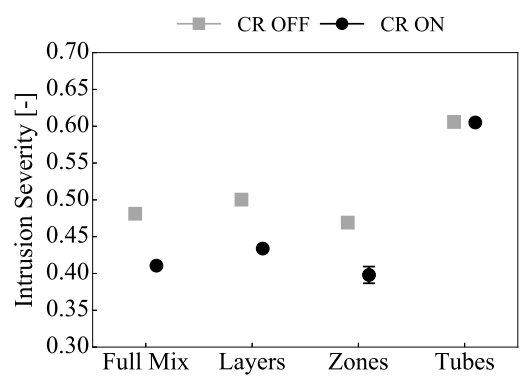

b) Effect of Conflict Resolution (CR)

Figure 11. Means and $95 \%$ confidence intervals of the intrusion severity metric

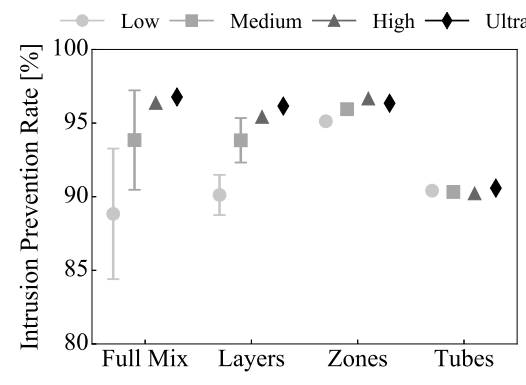

a) Effect of traffic demand (with conflict resolution)

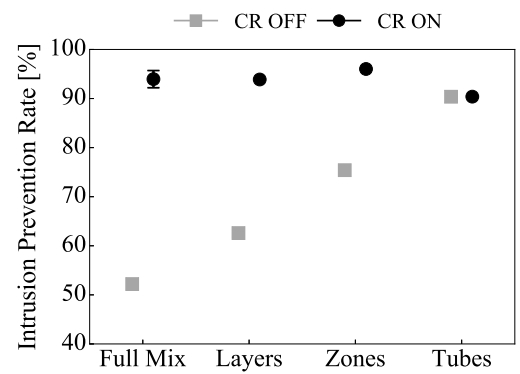

b) Effect of Conflict Resolution (CR)

Figure 12. Means and 95\% confidence intervals of the Intrusion Prevention Rate (IPR) metric

intrusions. Furthermore, the figure shows that intrusion severity is not significantly dependent on traffic demand. This suggests that intrusion severity is more a function of the selected CR algorithm than airspace structure. Due to the resolution maneuvers initiated by the MVP algorithm, intrusion severity was reduced when CR was enabled for Full Mix, Layers and Zones, see Figure 11b.

Figure 12 shows the results for the Intrusion Prevention Rate (IPR) metric, which measures the ability of a concept to solve conflicts without causing intrusions. For Full Mix, Layers and Zones, Figure 12a shows that IPR increases with traffic demand, while the rate of change of the metric appears to decrease with traffic demand and increasing airspace structure. This is because the rate of increase of intrusion number with traffic demand is less than that of 
conflict number, and the difference between the rates of change of conflicts and intrusions decreases with increasing demand and structure, compare Figures 8a and 9a.

More interestingly, Figure 12b shows that IPR is non-zero for all concepts with CR OFF. These conflicts, termed 'false conflicts' as they were resolved without intervention from the tactical CR algorithm, were likely to have been caused by the state based CD method used in this work. As this CD implementation relied on linear extrapolation of aircraft trajectories to predict conflicts, aircraft, which were turning or climbing/descending to follow concept dependent routing requirements, would trigger conflicts if they were momentarily in the projected paths of other aircraft. Figure 12b also shows that IPR increased with airspace structure for CR OFF, and is the highest for Tubes. This suggests that increasing traffic structure does improve the proportion of intrusions that can be avoided as a result of the prescribed routing of the more structured concepts, even though the absolute number of conflicts and intrusions were found to increase with structure and demand.

On the other hand, IPR was found to be very high (greater than 90\%) with CR ON for the three concepts that used tactical CR, see Figure 12b. Although false conflicts can also be expected for CR ON, the CP algorithm, which was also activated with tactical CR, prevented aircraft from turning into conflicts, thus avoiding conflicts of the type mentioned for the CR OFF case. Additionally, the similarity of this metric for Full Mix, Layers and Zones indicates that the relative performance of the MVP CR algorithm remains constant. However, as indicated above, the absolute number of intrusions has a greater bearing on the safety comparison between concepts.

\section{Efficiency}

Efficiency, measured using the work done metric, is shown in Figure 13. Here, a positive correlation between work done, level of airspace structure and traffic demand can be seen. The Full Mix concept displays the lowest work done, and is closely followed by the Layers concept. The difference between these two concepts can be traced back to the inefficient altitudes used by the Layers concept, whereas Full Mix used the most optimum horizontal and vertical flight paths to improve efficiency. The Tubes concept led to the highest work done, implying that aircraft flew significantly longer distances in this concept. For the Full Mix, Layers and Zones concepts, work done was found to be higher with

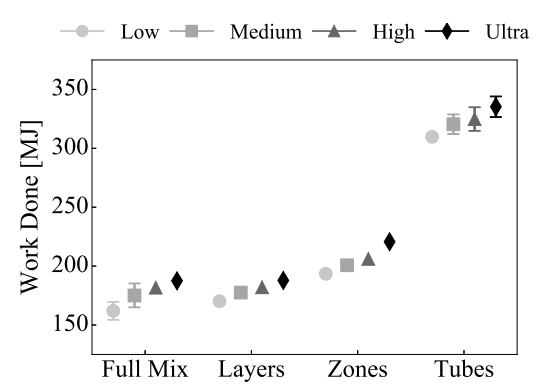

a) Effect of traffic demand (with conflict resolution)

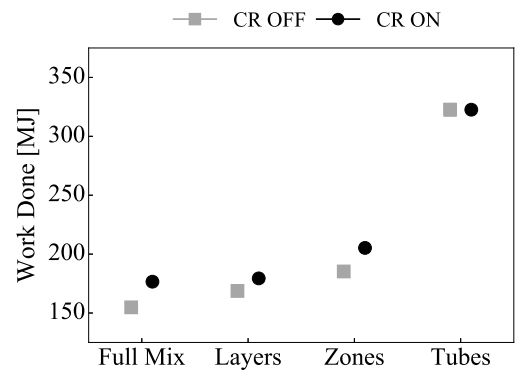

b) Effect of Conflict Resolution (CR)

Figure 13. Means and $95 \%$ confidence intervals of the work done metric 


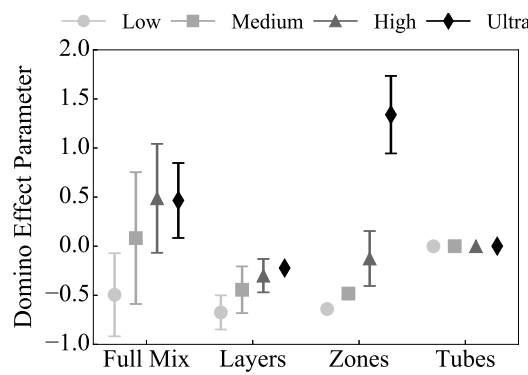

Figure 14. Means and $95 \%$ confidence intervals of the Domino Effect Parameter (DEP)

CR ON due to the extra distance flown during tactical CR maneuvers, see Figure 13b. These trends were also seen when comparing the distance traveled between concepts (not shown). Thus, the results strongly indicate that efficiency decreases with increasing airspace structure and density, as well as when tactical CR is used.

\section{Stability}

The stability of the airspace as result a of CR maneuvers is measured using the Domino Effect Parameter (DEP). A negative DEP implies a net stabilizing effect of tactical CR whereby conflict chain reactions are outweighed by those that are solved without pushing aircraft into secondary conflicts, whereas a positive value indicates the opposite, with conflict chain reactions causing airspace instability. The DEP for all concept-scenario combinations is pictured in Figure 14. Note that the DEP is consistently zero for Tubes as it did not use tactical CR. For the other three concepts, the DEP for the Low demand scenario is similar and negative. However at higher demand levels, the DEP increases to positive values for the Full Mix and Zones concepts. This suggests that the maneuvering room available to solve conflicts decreases rapidly with increasing airspace density for these two concepts, making it progressively more difficult to avoid intrusions without triggering additional conflicts. This is particularly true for the Zones concept which experienced a very large DEP increase between the High and Ultra demand scenarios, while for Full Mix, the DEP appears to settle at a value of approximately 0.5 .

Although the DEP also increased with demand for Layers, it remained negative for the range of densities considered in this work, see Figure 14. This suggests that the vertical segmentation of traffic used by the Layers concept is more able to prevent conflict propagation from occurring, and is better at assisting the MVP CR algorithm in solving the conflicts that do occur by reducing conflict angles and relative velocities between aircraft cruising at the same altitude, i.e., through the alignment of neighboring traffic. This result explains the reduction in the number of conflicts with CR ON, noted earlier for Layers (see Figure 8).

\section{Arrival Sequencing}

The number of consecutive arrivals with a time interval smaller than 60 seconds (equaling the CD look-ahead time), per destination, is shown in Figure 15a. This figure shows that the Tubes concept violated the 60 second threshold 

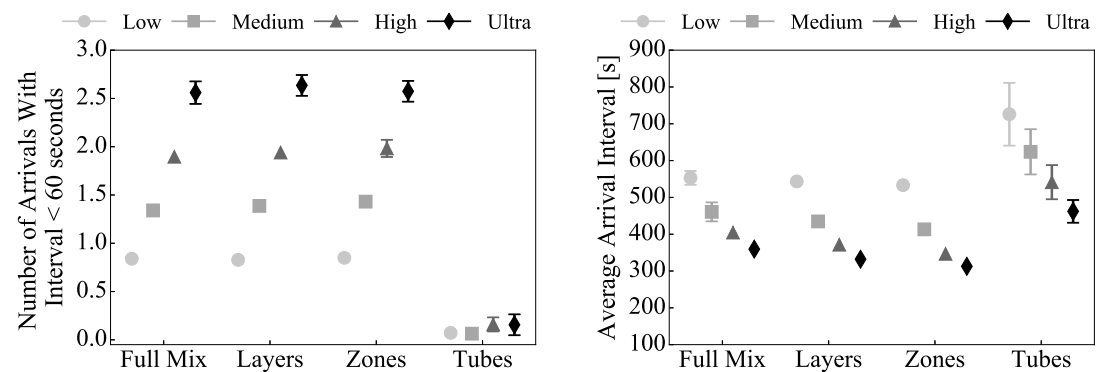

a) Number of arrivals with an interval less b) Average arrival interval between consecuthan 60 seconds tive landings

Figure 15. Means and $95 \%$ confidence intervals of the arrival sequencing between consecutive flights at each destination (with conflict resolution)

the least. This suggests that the time based separation used to separate traffic in the predefined tubes topology almost always ensured adequate separation at the runway. This is because all aircraft used the same descent rates below the experiment volume. Hence a runway conflict could only occur only if two or more aircraft attempted to land at the same runway at the same time from different nodes in the topology (a scenario with a low probability given the reduced traffic volume accommodated by Tubes). The other three concepts, however, resulted in up to 2.6 aircraft arriving with insufficient spacing at the runway for the Ultra demand scenario. Although this is partly due to the higher traffic volumes handled by these concepts, this result shows that some form of arrival metering may be necessary for Full Mix, Layers and Zones. But, as the average arrival interval between aircraft is always greater than 300 seconds for these three concepts, see Figure 15b, arrival sequencing can likely be managed by varying the speeds of conflicting aircraft during the final descent. Given the high average arrival intervals for all concepts, the different ways of structuring en-route airspace are not expected adversely impact arrival procedures.

\section{Structural Complexity}

Figure 16 displays the results for the Structural Complexity $(S C x)$ metric, normalized with respect to the total number of flights simulated per concept. Here, high values corresponds to traffic situations that are more difficult to control, and those that are more sensitive to uncertainties. Figure 16a shows that $S C x$ followed a similar trend to the number of conflicts and intrusions, with a clear distinction between the two less structured concepts, which display a linear growth of $S C x$ with demand, when compared to the two more structured concepts, which resulted in a quadratic increase of $S C x$ with demand. This strongly suggests that little structuring of airspace, as used by Full Mix and Layers, results in traffic patterns that are easier to control than those produced by the predetermined routes of the Zones and Tubes concepts.

The pre-planned routes used by the Tubes concept were expected to result in the lowest complexity. In fact, the absolute $S C x$ values were the lowest for Tubes. However, when the results were normalized, the contribution of each flight to $S C x$ was found to be much higher for Tubes than for the other concepts. The high complexity of the Zones 


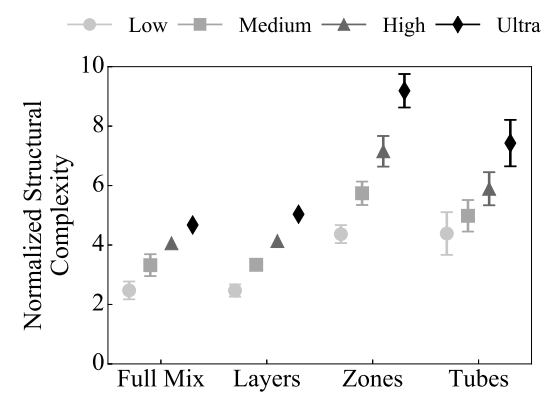

a) Effect of traffic demand (with conflict resolution)

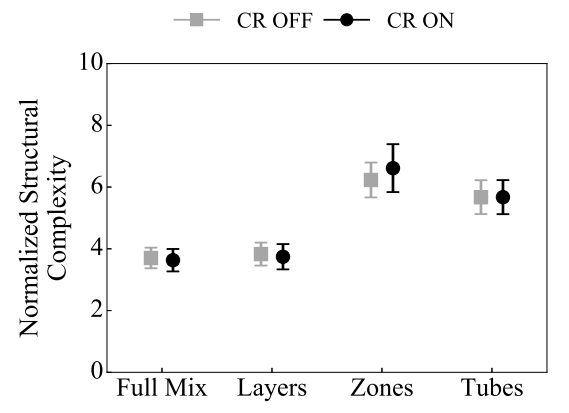

b) Effect of Conflict Resolution (CR)

Figure 16. Means and $95 \%$ confidence intervals of the normalized Structural Complexity $(S C x)$ metric

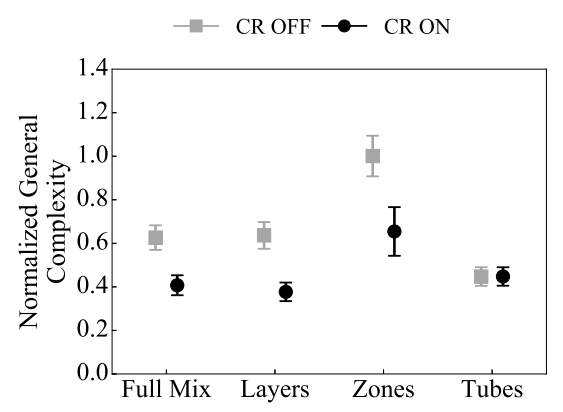

Figure 17. Means and $95 \%$ confidence intervals of the effect of Conflict Resolution (CR) on the normalized general complexity metric

concept can be traced to traffic convergence at the intersections between ring and radial zones.

As expected, no differences in $S C x$ between CR ON and CR OFF were found for the Tubes concept, see Figure 16b. However, the overlapping error-bars for the Full Mix, Layers and Zones concepts indicate that $S C x$ was not significantly affected by CR maneuvers. This result can be explained by the fact that the computation of the SCx metric considers all possible trajectory realizations/evolutions between aircraft pairs within a predefined spatiotemporal window, negating the effect of the specific resolution maneuvers used to avoid intrusions. Therefore, it can be concluded that the $S C x$ metric is not suitable to analyze the effect of CR maneuvers on intrinsic complexity.

To gain more insight on the effect of CR on complexity, the influence of CR maneuvers on the general complexity metric, described by eq. 5, is displayed in Figure 17. Here, it can be seen that CR ON reduced the general complexity metric for the Full Mix, Layers and Zones concepts, indicating that CR improves the controllability of a given traffic situation, as expected.

\section{Noise Pollution}

Noise footprints for all four concepts are displayed in Figure 18. This figure was created using data from a simulation for the 'Ultimate' demand volume and with a converging demand pattern, see Section III.B.2. Figures 18a and 18b show that Full Mix and Layers resulted in very similar noise loads on the ground, consisting of a high intensity noise 


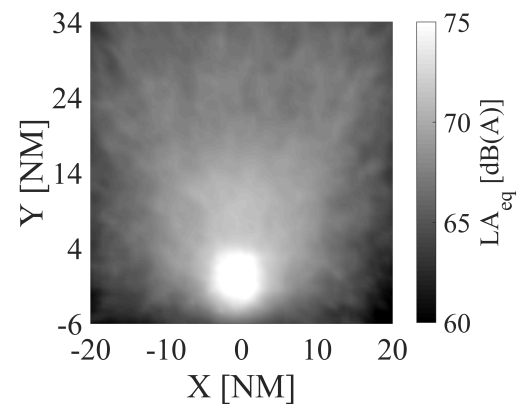

a) Full Mix

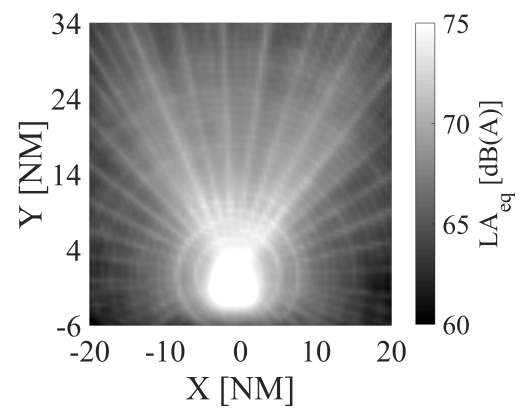

c) Zones

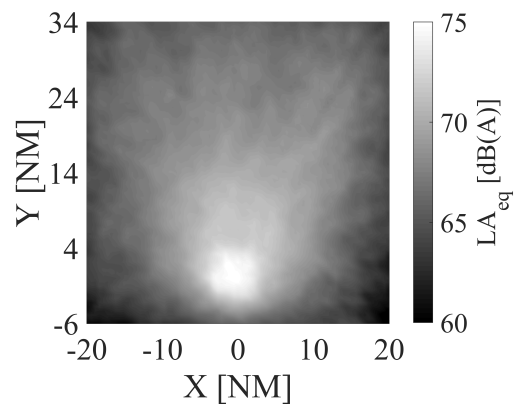

b) Layers

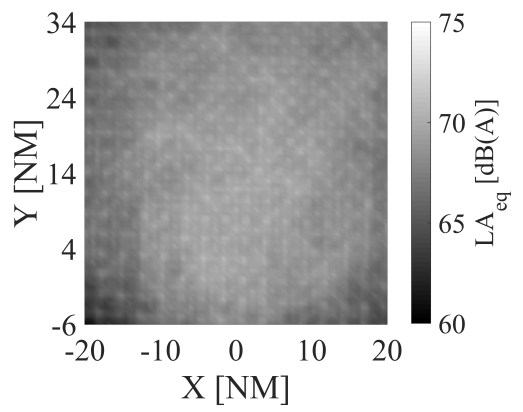

d) Tubes

Figure 18. Noise footprints for an 'Ultimate' demand scenario with a converging traffic demand pattern

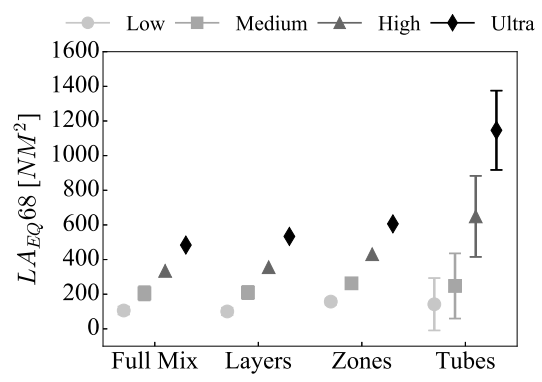

a) Effect of traffic demand (with conflict resolution)

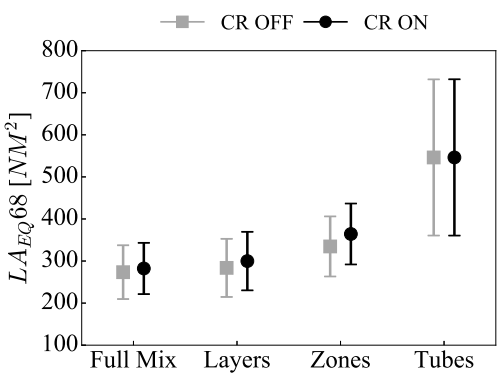

b) Effect of Conflict Resolution (CR)

Figure 19. Means and 95\% confidence intervals of the area encapsulated by the $L A_{e q}$ noise metric for iso-contour lines at $68 \mathrm{~dB}$

load at the main convergence point at the bottom of the simulation area, that decayed with increasing distance from that point. In contrast, the Zones and Tubes concepts resulted in footprints that clearly depicts the structuring of traffic used by these two concepts. For the Zones concept, Figure 18c shows higher noise levels along the ground projections of the predefined ring and radial zones. This suggests that the Zones concept may allow for easier mitigation of noise pollution by defining its topology along less inhabited areas.

The Tubes concept is shown to have distributed sound evenly due to its grid-like structure, see Figure 18d. Furthermore, Tubes appears to have caused the lowest noise impact on the ground and was the only concept without a high intensity noise load at the convergence point. However, these trends are related to the lower traffic volume that could be accommodated by the Tubes concept, see Figure 7a. Unlike the other metrics discussed above, a linear nor- 
malization with respect to the total number of flights could not be used as noise is measured in $\mathrm{dB}$, a logarithmic unit. This complicates a direct comparison with the Tubes concept in relation to noise pollution.

In addition to the noise footprints discussed above, the LAeq metric is used to quantitatively study the noise impact by computing the area encapsulated by iso-contour lines at the $68 \mathrm{~dB}(\mathrm{~A})$ level. Figure 19a shows that noise pollution at $68 \mathrm{~dB}(\mathrm{~A})$ is quite similar for Full Mix, Layers and Zones. On the other hand, the Tubes concept, which caused the lowest total noise impact on the ground, led to the largest area at the $68 \mathrm{~dB}(\mathrm{~A})$ level for the higher demand scenarios. This suggests that the total noise on the ground would have been the highest for the Tubes concept had it been able to meet the required traffic demand. As expected, CR ON led to a slightly higher noise impact for the Full Mix, Layers and Zones concepts, see Figure 19b, as resolution maneuvers increased flight distances for these three concepts.

\section{B. Non-Nominal Experiment}

As stated earlier, the purpose of the non-nominal experiment is to compare the relative robustness of the four airspace concepts when subjected to increasing numbers of rogue aircraft. Since rogue aircraft primarily affect safety metrics, the following paragraphs discuss the number of conflicts and intrusions between rogue and 2.7 million normal aircraft that were logged during this experiment.

Figures 20 and 21 display the number of conflicts and intrusions per flight with rouge aircraft alone, i.e., only safety incidents between normal and rouge aircraft were counted for these two figures. Here it can be seen that increasing the number of rogue aircraft also increases the number of conflicts and intrusions for all concepts. Further analysis indicated that the nonlinear increase in the number of rogue aircraft only led to a linear increase in the number of conflicts and intrusions for all concepts, suggesting that all concepts are more robust than initially expected. Nonetheless, Figures 20a and 21a show that the safety of the Tubes concept is considerably more affected by rouge aircraft than it is for the Full Mix, Layers and Zones concepts.

As the trajectories of rogue aircraft were not known in advance, aircraft in the Tubes concept used the MVP CR algorithm to avoid intrusions with rogue aircraft alone. Since the tube topology specifies both the horizontal and vertical flight profiles, only speed resolution maneuvers were possible. Figures $20 \mathrm{~b}$ and $21 \mathrm{~b}$ shows that these

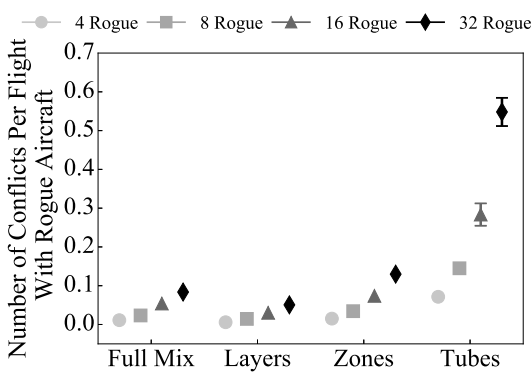

a) Effect of number of rogue aircraft (with conflict resolution)

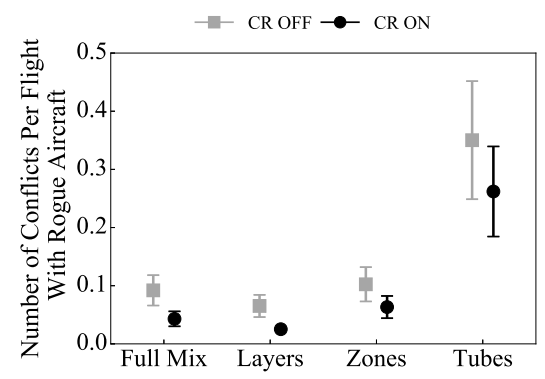

b) Effect of Conflict Resolution (CR)

Figure 20. Means and $95 \%$ confidence intervals of the number of conflicts per flight with rogue aircraft alone 


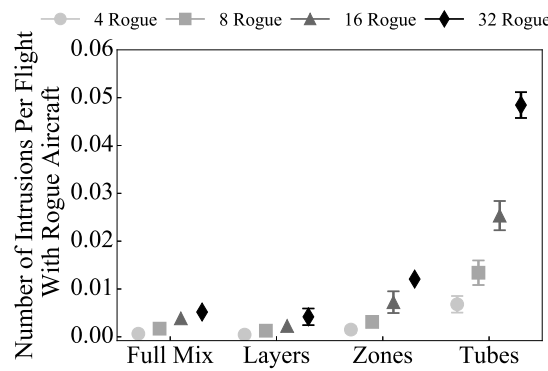

a) Effect of number of rogue aircraft (with conflict resolution)

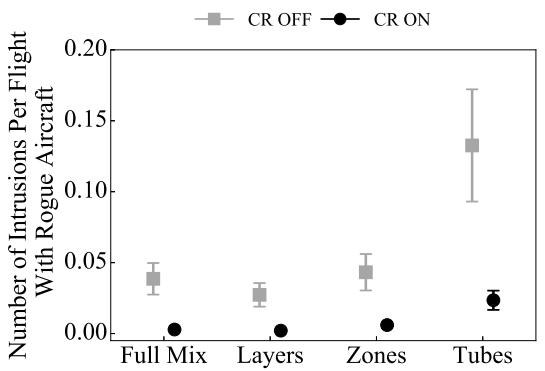

b) Effect of Conflict Resolution (CR)

Figure 21. Means and $95 \%$ confidence intervals of the number of intrusions per flight with rogue aircraft alone

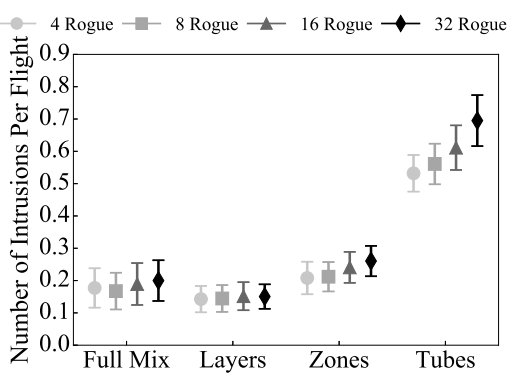

Figure 22. Means and $95 \%$ confidence intervals of the total number of intrusions per flight for the non-nominal experiment (with conflict resolution)

resolutions did reduce the number of conflicts and intrusions with rogue aircraft for Tubes. As expected, CR ON also improved the safety of the other three concepts against rogue aircraft.

The above result may suggest that tactical CR can be used to complement the safety of the pre-planned Tubes concept. However, closer inspection of the simulation data indicated that CR maneuvers made it more difficult for conflict resolving (normal) aircraft to meet the specified Required Time of Arrival (RTA) at waypoints along preplanned routes. This in turn resulted in additional intrusions between normal aircraft, that were avoided when rogue aircraft were not included in the simulations. In fact, the resulting break-down of the pre-planned routes caused the highest total number of intrusions per flight for the Tubes concept, while for the three less structured concepts, the total number of intrusions was not affected by the number of rogue aircraft, see Figure 22. Therefore the simple addition of tactical CR is not guaranteed to improve the robustness of the Tubes concept. To improve robustness and safety, larger margins could be applied to time constraints at waypoints along a route. However, this would further decrease the efficiency of the Tubes concept, see Figure 13a.

\section{Discussion}

In this paper, four en-route airspace concepts of increasing structure, named Full Mix, Layers, Zones and Tubes, were compared using two fast-time simulation experiments that involved over eleven million flights. These simulations focused on the degree of structuring needed to maximize capacity for decentralized separation, and investigated 
whether the optimal mode of structuring depended on traffic density. The simulations also studied the robustness of the different structuring methods to non-nominal events. Here, capacity was inferred from the effect of multiple traffic demand scenarios on safety, efficiency, stability, arrival sequencing, structural complexity and noise pollution metrics, while robustness was assessed from the influence of increasing numbers of rogue aircraft on safety metrics.

As indicated previously, the goal of this work is not to propose an operationally ready airspace design, but to focus on how the level of structuring affects airspace capacity for decentralization. In relation to this specific goal, and considering the results of all metrics in unison, it can be concluded that some limited structure in the vertical dimension, as demonstrated by the Layers concept, can be beneficial in terms of capacity. This is because traffic demand, in addition to varying in time, displays no predominant patterns in the horizontal dimension for decentralization. Therefore, a strict horizontal structuring of airspace can cause a mismatch between the imposed structure and the demand pattern, as for the Zones and Tubes concepts. This in turn caused artificial bottlenecks at the intersection points of their predefined topologies, reducing overall performance. On the other hand, the vertical airspace segmentation used by the Layers concept dispersed traffic vertically, and grouped traffic by similarity of travel direction. This reduced the chance of conflicts by lowering relative velocities between cruising aircraft when compared to the completely unstructured Full Mix concept, without unduly affecting efficiency, structural complexity or noise pollution metrics, as direct horizontal routes were still possible with Layers. These conclusions were not expected as previous research had focused primarily on either fully structured or fully unstructured airspace designs.

For the range of densities considered in this work, the results of the nominal simulations also indicate that the optimum method of structuring is independent of traffic density. In fact, the results show a clear distinction between the two less structured and the two more structured concepts; while performance degraded with increasing density for all concepts, it did so at a much higher rate for Zones and Tubes. In particular, the safety of the Zones and Tubes concepts deteriorated rapidly for the higher densities, indicating that the airspace had become saturated for these two highly structured concepts. When considering the fact that route planning was more computationally intensive for Zones and Tubes, the additional constraints imposed on traffic by these two concepts did not translate into benefits in terms of capacity.

The clear distinction between the two more and two less structured concepts was not, however, found for the results of the non-nominal simulations. In that experiment, the safety of the Full Mix, Layers and Zones concepts was not significantly affected by rogue aircraft. This suggests that tactical conflict resolution, which was used natively by these three concepts to resolve conflicts between nominal aircraft, could also effectively compensate for noncooperative aircraft, without adversely affecting capacity. This was not the case for the Tubes concept. Here, the uncertainties caused by rogue aircraft made it difficult for nominal aircraft to adhere to time constraints at waypoints along a route. The resulting break down of the pre-planned space-time routes used by Tubes to separate aircraft caused a large number of unintended conflicts and intrusions. The performance of Tubes was also affected by wind for the same reason. While all concepts were negatively influenced by uncertainties, the current results show that the safety 
of highly structured and planned airspace concepts is particularly vulnerable to variations between the intended and actual flight trajectories.

For many metrics, the Full Mix and Layers concepts exhibited very similar behavior. The similarity between these two concepts was highlighted by their noise footprints, which were indistinguishable. The only difference between these two concepts is the method of altitude selection; aircraft in Full Mix used the most fuel-efficient altitude, while for Layers, altitude was dependent on the direction to the destination. Therefore, Full Mix was expected, and was also found, to be slightly more energy efficient. However, the similarity in efficiency between these two concepts may be a result of the short cruising distances used in this work, and longer distances may further increase the efficiency differences between Full Mix and Layers.

Although the vertical structuring used by Layers reduced efficiency relative to the unstructured Full Mix concept, it led to the highest stability of all four structuring options. This high stability was reflected by the negative Domino Effect Parameter (DEP) values logged for all densities in the Layers concept. A negative DEP indicates a reduction in the number conflicts when the tactical Conflict Resolution (CR) algorithm is enabled, even though CR maneuvers increase flight distances and, correspondingly, the probability of encountering other aircraft. It is hypothesized that a negative DEP is caused by the alignment of neighboring traffic, and the consequent reduction of relative velocities that aids CR performance, a behavior that is further amplified by the limited heading ranges available to cruising aircraft in the Layers concept. Although negative DEPs were also found for Full Mix and Zones at low demand levels, at higher densities, these modes of structuring transitioned to positive DEP vales, indicating a greater degradation of airspace stability with density. Hence, the stability analysis indicates that the altitude constraints utilized by Layers can accommodate even higher densities than what was considered in this work, without significantly reducing safety or efficiency. This reiterates the notion that a limited degree of vertical structuring can be beneficial for the capacity and robustness of decentralized airspace.

It should be noted that the results of this study are, to some degree, sensitive to the specific parameter settings selected for the concepts. However, given the magnitude of the differences in all of the results, as well as the consistency between results, it is unlikely that the overall trends are affected by different settings; capacity for decentralization was found to improve when structural constraints did not affect the horizontal path of aircraft. This conclusion is most applicable for traffic scenarios without any distinct horizontal patterns. For traffic demand cases with discernible horizontal patterns, such as for current hub-and-spoke operations, airspace concepts that permit flexible routing in the horizontal direction are also expected to perform well, as such structuring would not conflict with any demand pattern. Nonetheless, it may be possible to tailor the topologies of more structured concepts to match such scenarios. 


\section{Conclusions}

This work investigated the degree of structuring needed to maximize capacity for decentralized en-route airspace. To this end, four decentralized en-route airspace concepts of increasing structure were compared using fast-time simulations. For the studied densities, the following conclusions can be drawn:

- Capacity benefits when the horizontal path of aircraft is not over-constrained. This is because traffic demand displays no predominant patterns in the horizontal dimension, for decentralization.

- Capacity is maximized when vertical constraints are used to separate traffic with different travel directions at different flight levels. This mode of structuring improved performance over completely unstructured airspace by decreasing relative velocities between aircraft cruising at the same altitude, while allowing direct horizontal routes. The reduced relative velocities also increased the stability of the airspace to tactical conflict resolutions.

- Conversely, structuring modes that imposed horizontal constraints caused a convergence of traffic at the intersections of structural elements. These traffic concentrations reduced overall performance for such concepts.

- The optimum method of structuring was found to be independent of density. Capacity generally benefited from a reduction of structural constraints.

- Robustness to uncertainties is significantly reduced when decentralization using time based separation is combined with a predefined and fixed three dimensional route structure.

\section{Acknowledgments}

This research received funding from the Seventh Framework Programme of the European Commission under grant number 341508 (Metropolis). The authors would like to thank Pim van Leeuwen (NLR) for assisting with the design of the Tubes concept, Roalt Aalmoes (NLR) for his work on metrics definition, Oliver Schneider and Stefan Kern (DLR) for their efforts towards the design of the Layers concept, Daniel Delhaye (ENAC) for his insights on air traffic complexity measurement, Georges Mykoniatis (ENAC) for helping with project management tasks and Marieke Suijkerbuijk (NLR) for aiding with simulation implementation tasks.

\section{References}

[1] Lee, P. U., Mercer, J., Gore, B., Smith, N., Lee, K., and Hoffman, R., "Examining airspace structural components and configuration practices for dynamic airspace configuration," AIAA Guidance, Navigation, and Control Conference, AIAA2008-7228, 2008, pp. 18-21.

[2] Doble, N. A., Hoffman, R., Lee, P. U., Mercer, J., Gore, B., Smith, N., and Lee, K., "Current airspace configuration practices and their implications for future airspace concepts," AIAA Aviation Technology, Integration and Operations (ATIO) Conference, AIAA-2008-8936, Anchorage, 2008. 
[3] Nolan, M., Fundamentals of Air Traffic Control, Chapter 1 History of ATC, Delmar Cengage Learning, 5th ed., 2010.

[4] Magill, S. A. N., "Effect of Direct Routing on ATC Capacity," USA/Europe Air Traffic Management R\&D Seminar, Orlando, 1998.

[5] Eby, M. S., “A Self-Organizational Approach for Resolving Air Traffic Conflicts," Lincoln Laboratory Journal, Vol. 7, No. 2, 1994, pp. 239-254.

[6] Hoekstra, J. M., van Gent, R. N. H. W., and Ruigrok, R. C. J., "Designing for safety: the free flight air traffic management concept,” Reliability Engineering \& System Safety, Vol. 75, No. 2, Feb. 2002, pp. 215-232.

[7] Dell'Olmo, P. and Lulli, G., "A new hierarchical architecture for Air Traffic Management: Optimisation of airway capacity in a Free Flight scenario,” European Journal of Operational Research, Vol. 144, No. 1, Jan. 2003, pp. 179-193.

[8] SESAR Consortium, “The Concept of Operations at a glance,” Tech. rep., Single European Sky, 2007.

[9] Joint Planning and Development Office, “Concept of operations for the Next Generation Air Transportation System,” Tech. rep., FAA, June 2007.

[10] Kuchar, J. K. and Yang, L. C., "A Review of Conflict Detection and Resolution Modeling Methods," IEEE Transactions on Intelligent Transportation Systems, Vol. 1, 2000, pp. 179-189.

[11] Ballin, M., Hoekstra, J., Wing, D., and Lohr, G., "NASA Langley and NLR Research of Distributed Air/Ground Traffic Management," AIAA Aircraft Technology, Integration, and Operations (ATIO) Conference, AIAA-2002-5826, American Institute of Aeronautics and Astronautics, 2002.

[12] Krozel, J., Peters, M., Bilimoria, K., Lee, C., and Mitchell, J., "System performance characteristics of centralized and decentralized air traffic separation strategies," USA/Europe Air Traffic Management R\&D Seminar, 2001.

[13] Klooster, J., Torres, S., Earman, D., Castillo-Effen, M., Subbu, R., Kammer, L., Chan, D., and Tomlinson, T., “Trajectory synchronization and negotiation in Trajectory Based Operations," IEEE/AIAA Digital Avionics Systems Conference (DASC), 2010, pp. 1.A.3-1-1.A.3-11.

[14] Prevot, T., Battiste, V., Palmer, E., and Shelden, S., "Air traffic concept utilizing 4D trajectories and airborne separation assistance," AIAA Guidance, Navigation, and Control Conference, AIAA-2003-5770, 2003.

[15] Wichman, K., Lindberg, L., Kilchert, L., and Bleeker, O., "Four-Dimensional trajectory based air traffic management," AIAA Guidance, Navigation, and Control Conference, AIAA-2004-5413, 2004, p. 11.

[16] International Civil Aviation Organization, “Annex 2, Rules of the Air,” Tech. rep., July 2005.

[17] Bussink, F., Hoekstra, J., and Heesbeen, B., "Traffic manager: a flexible desktop simulation tool enabling future ATM research,” IEEE Digital Avionics Systems Conference (DASC), Vol. 1, IEEE, 2005, pp. 3.B.4 - 31-10.

[18] Nuic, A., Poles, D., and Mouillet, V., "BADA: An advanced aircraft performance model for present and future ATM systems," International Journal of Adaptive Control and Signal Processing, Vol. 24, No. 10, 2010, pp. 850-866.

[19] Hart, P., Nilsson, N., and Raphael, B., "A Formal Basis for the Heuristic Determination of Minimum Cost Paths,” IEEE Transactions on Systems Science and Cybernetics, Vol. 4, No. 2, July 1968, pp. 100-107. 
[20] Hoekstra, J. M., Designing for safety: the free flight air traffic management concept, PhD Dissertation, Delft University of Technology, Delft, Nov. 2001.

[21] Sunil, E., Hoekstra, J., Ellerbroek, J., Bussink, F., Nieuwenhuisen, D., Vidosavljevic, A., and Kern, S., "Metropolis: Relating Airspace Structure and Capacity for Extreme Traffic Densities," USA/Europe Air Traffic Management R\&D Seminar, Lisbon, 2015.

[22] Bilimoria, K., Sheth, K., Lee, H., and Grabbe, S., "Performance evaluation of airborne separation assurance for free flight," AIAA Guidance, Navigation and Control Conference, AIAA-2000-4269, 2000.

[23] Delahaye, D. and Puechmorel, S., "Air traffic complexity: towards intrinsic metrics," USA/Europe Air Traffic Management $R \& D$ Seminar, 2000.

[24] Vidosavljevic, A., Delahaye, D., Sunil, E., Bussink, F., and Hoekstra, J.M., "Complexity Analysis of the Concepts of Urban Airspace Design for Metropolis Project," ENRI International Workshop on ATM/CNS, 2015.

[25] Ruijgrok, G. J., Elements of Aviation Acoustics, Chapter 9 Noise Measures, Delft University Press, 2004.

[26] Boeker, E. R., Dinges, E., He, B., Fleming, G., Roof, C. J., Gerbi, P. J., Rapoza, A. S., and Hemann, J., "Integrated Noise Model (INM) Version 7.0,” Tech. Rep. FAA-AEE-08-01, Jan. 2008.

[27] Arntzen, M., Aalmoes, R., Bussink, F., Sunil, E., and Hoekstra, J. M., "Noise computation for future urban air traffic systems," Inter-Noise Congress: Implementing Noise Control Technology, 2015. 